\title{
IoT Dataset Generation Framework for Evaluating Anomaly Detection Mechanisms
}

\author{
Andreas Meyer-Berg \\ Technische Universität Darmstadt \\ Darmstadt, Hessen, Germany \\ andreas.meyer-berg@stud.tu-darmstadt.de \\ Leon Böck \\ Technische Universität Darmstadt \\ Darmstadt, Hessen, Germany \\ boeck@tk.tu-darmstadt.de
}

\author{
Rolf Egert \\ Technische Universität Darmstadt \\ Darmstadt, Hessen, Germany \\ egert@tk.tu-darmstadt.de \\ Max Mühlhäuser \\ Technische Universität Darmstadt \\ Darmstadt, Hessen, Germany \\ max@tk.tu-darmstadt.de
}

\begin{abstract}
Machine learning based anomaly detection mechanisms are a promising tool to detect and protect networks from previously unknown attacks. The quality of those mechanisms strongly depends on the availability of large amounts of data for their training and evaluation. However, suitable datasets are scarce, as they are rarely shared by those who possess them. This impedes progress in the development and deployment of sophisticated machine learning mechanisms. This paper aims to accelerate this thwarted development process by introducing a network simulation framework for training-data generation and evaluation of data-driven mechanisms, like anomaly detection approaches. The framework enables training, testing, and evaluating data-driven approaches in a safe and extensible environment prior to their deployment in real-world systems. We showcase the capabilities of the framework in a case study. For this, a smart home network is modeled and simulated within the framework. The generated data is used to train an anomaly detection approach, which is then used to detect various anomalies introduced by attacks on the network. This ability to train and evaluate data-driven algorithms within the framework allows users to accelerate the otherwise time-consuming cycle of deploying, modifying, and re-training in live environments, which ultimately advances the development of novel anomaly detection approaches.
\end{abstract}

\section{CCS CONCEPTS}

- Security and privacy $\rightarrow$ Intrusion detection systems; Network security; • Computing methodologies $\rightarrow$ Discrete-event simulation.

\section{KEYWORDS}

Network simulation, network security, IoT

ACM Reference Format:

Andreas Meyer-Berg, Rolf Egert, Leon Böck, and Max Mühlhäuser. 2020. IoT Dataset Generation Framework for Evaluating Anomaly Detection

Permission to make digital or hard copies of part or all of this work for personal or classroom use is granted without fee provided that copies are not made or distributed for profit or commercial advantage and that copies bear this notice and the full citation on the first page. Copyrights for third-party components of this work must be honored.

For all other uses, contact the owner/author(s).

ARES 2020, August 25-28, 2020, Virtual Event, Ireland

(C) 2020 Copyright held by the owner/author(s).

ACM ISBN 978-1-4503-8833-7/20/08.

https://doi.org/10.1145/3407023.3407036
Mechanisms. In The 15th International Conference on Availability, Reliability and Security (ARES 2020), August 25-28, 2020, Virtual Event, Ireland. ACM, New York, NY, USA, 6 pages. https://doi.org/10.1145/3407023.3407036

\section{INTRODUCTION}

Proactive security mechanisms such as firewalls or anti-virus scanners have been circumvented repeatedly in the past and will be bypassed again by novel and more sophisticated threats. This problem increased even more with the emergence of the Internet of Things (IoT), where security mechanisms are limited by scarce resources [17] or simply neglected by the developers [3]. Reactive security mechanisms, such as Intrusion Detection Systems (IDSs), can alleviate this situation by establishing a second line of defense.

A considerable trend in the field of IDSs focuses on machine learning techniques for detecting anomalies [24]. These approaches aim at learning a model of normality, which is then used to classify whether observed data traffic is considered abnormal or not. In the context of IoT, anomaly detection can not only be applied to the network traffic itself, but also to the application layer data of sensors or actuators [17]. Applying anomaly detection on this layer can detect intrusions that may be missed if only the lower layers of network traffic are analyzed. As an example, a manipulated thermostat may show no irregularities on lower layers, whereas the actual temperature readings indicate an anomaly.

The development and deployment of machine learning based IDS approaches is a challenging task. Three prominent challenges are: (a) scarcity of suitable training data, (b) the time that is required for re-training approaches to adapt them to the peculiarities of the target network, and (c) limited possibilities to evaluate anomaly detection approaches in the target networks and assess their corresponding (potentially threatening) security implications [7].

One solution to these challenges is a network simulation framework. Such a framework could generate infinite amounts of data (a) and quickly evaluate and re-train mechanisms (b) in a safe virtual environment before deploying them in the target networks (c).

Various established network simulators exist that are capable of modeling and simulating protocols and networks [12]. However, no known frameworks combine network simulation and a userfriendly evaluation of algorithms based on application-layer data. In this context, we propose a framework for simulating network communication to support the development and evaluation of algorithms that are based on application-layer data. The framework 
enables quick prototypical deployment of mechanisms and simplifies their modification by supporting adjustments and re-training within the framework, thereby, avoiding frequent redeployment into a real network. The main contributions are as follows: 1) An extensible framework for modeling and simulating protocols and networks, 2) bulk dataset generation for training and testing of algorithms based on application-layer data, and 3) showcasing the framework's abilities using a simple anomaly detection approach.

The remaining paper is structured as follows: In Section II related works are presented. Section III, introduces the simulation framework and its main capabilities. Subsequently, Section IV evaluates the framework in a case study, before we conclude in Section V.

\section{RELATED WORK}

Within this section, related work on network simulators, traffic generators, and smart home simulators is presented.

\subsection{Network Simulators}

The simulation of computer networks has been studied extensively with a multitude of programs and approaches. This section aims to describe the most significant approaches and their extensions. The discrete network simulators $n s-2$ [15] and $n s-3$ [18] support the simulation of various protocols, routing algorithms, and network types, e.g., wired/wireless networks. Moreover, they allow importing userdefined protocols and devices and provide user interface support. Chengze Fan et al. [9] proposed NS4, an extension of ns-3, which adds support of the $\mathrm{P} 4$ [4] programming language. The P4 programming language can program the data plane in software-defined networks and simplifies the programming of switches. Gns-3 [26] is an open-source tool with a focus on emulating hardware. It allows simulating small networks, emulation of Cisco and other hardware, PCAP creation, and interactions with real networks. The combination of the discrete OMNeT++ [25] simulator with the INET [25] framework, makes it a highly flexible option for network simulation and traffic generation. Aside from open-source simulators, the following commercial options exist. NetSim [23] is a discrete-event network simulator, which enables simulation of various network types and protocols. It offers a user-friendly interface and supports various IoT settings and protocols. Furthermore, NetSim supports interfaces to other tools like Matlab and Wireshark. The Simple IoT Simulator [20] allows to simulate and visualize IoT networks. It offers multiple application-layer protocols like MQTT, COAP, and HTTP as well as underlying protocols such as IPv4, and IPv6. Additionally, the configuration of small scripts is supported and utility functions for capturing and replicating traffic of real devices are provided. Lastly, the Cisco Packet Tracer [1] offers a proprietary network simulator, for educational purposes of the Cisco Networking Academy. It offers a user interface to build, configure, and experiment with networks and corresponding protocols, network types, and technologies.

\subsection{Traffic Generators}

The open-source traffic generator Ostinato [10] allows packet generation, offers a user interface and the developers describe it as
"Wireshark in Reverse". Further features include: fine-grained control over the stream rates, receive/transmit statistics, PCAP creation/editing, and support of protocols like IPv4, UDP, and HTTP. The Intrusion Detection Dataset Toolkit (ID2T) [8] can generate datasets for the evaluation of IDS systems. Given an input PCAP file, ID2T can inject various network attacks emphasizing on resembling real attack traffic. The output is another PCAP file containing the attack traffic and a label file indicating the injected traffic.

\subsection{Smart Home Simulators}

In their survey, Synnott et al. [22] suggest two categories for smart home simulators: model-based approaches and interactive approaches. Model-Based Approaches employ a model of the smart home and especially its different activities, which determine the probability, order, and time-span of events. Tools in this family, include SIMACT [5], Persim [11], and DiaSim [6]. Interactive approaches allow interacting with the model during the simulation through live or avatar-based interaction with virtual devices, such as turning on an appliance. The most popular approaches are UbiReal [16], and OpenSHS [2]. However, the main focus of the presented smart home simulators is on activities and human interaction within smart homes and less on network and data processing. In contrast to the previously presented work, our proposed framework poses an extensible simulation and evaluation environment for data-driven algorithms like machine learning based anomaly detection. For this, the framework offers a unique combination of traffic generation, network simulation, and live-evaluation of algorithms in a virtual environment. These combined capabilities support and improve the development of data-driven approaches and speed-up their deployment in real-world scenarios.

\section{FRAMEWORK DESCRIPTION}

This section introduces the discrete-event simulation framework ${ }^{1}$. For that, the network model, user interface, the extensible design, and simulation capabilities are introduced in that order.

\subsection{Network Model}

The network model offers a high-level representation of a layerednetwork stack. Figure 1 shows the general design concept of the network model, which can be subdivided into five main layers, namely Smart Devices, Ports, Protocols, Connections, and Links. Smart Devices represent and mimic devices such as sensors, actuators, and collectors, which communicate in a network environment. A Smart Device stores an internal state, like sensor information, configuration details, and multiple ports. The framework offers different interfaces for sensors or collectors to generate and receive data, to allow easy extension with new sensors and collectors. For instance, BoolCollectorDevice and FloatSensorDevice implement the previously mentioned interfaces to generate and receive boolean or float values and exchange them using a protocol like MQTT.

The Port class models networking ports, which represent endpoints of Connections. A Port stores time-response characteristics and additional attributes like affiliation to a Connection. A Port has the states CLOSED, OPEN, and SENDING. A CLOSED port does not respond to any messages, OPEN ports reply to incoming messages

\footnotetext{
${ }^{1}$ https://git.tk.informatik.tu-darmstadt.de/SPIN/IoTDatasetGenerationFramework
} 


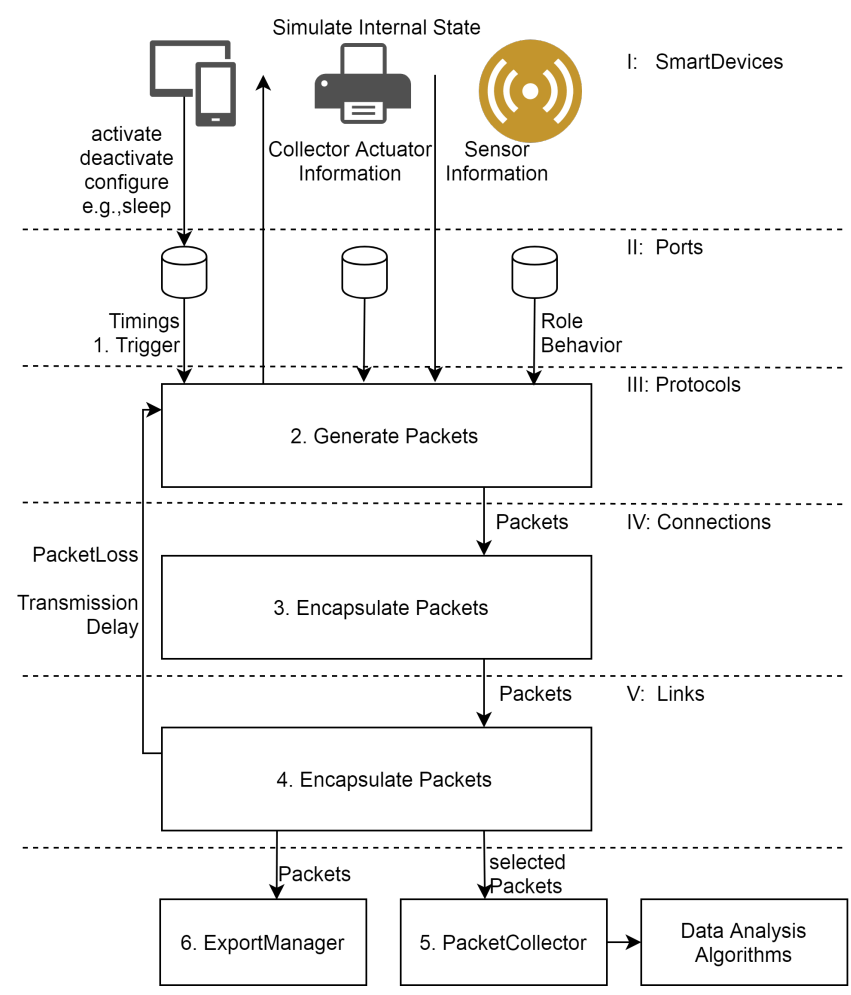

Figure 1: Abstract representation of the network model

and SENDING ports extend OPEN ports by actively sending messages. To avoid static traffic behavior, sending intervals and delays are modeled using constant, Gaussian and Poisson distributions, which can be freely configured and extended by the user.

Protocols are used to model application-layer protocols like MQTT. Values exchanged by Smart Devices are embedded into the corresponding packets by the Protocol modules. A Protocol module defines the role of the Smart Device. In the case of MQTT the roles are broker, subscriber, publisher, and subscriber \& publisher. These roles enable the Protocol module to automatically process the packets further. The Protocol adds packet loss and transmission delays, to further increased the resemblance of real world traffic. Additionally, Protocols can provide predefined response packets to avoid re-implementation in internal logic of each Smart Device. Transport or network-layer protocols are modeled by the Connection class. A Connection encapsulates the packets generated by a higher-level Protocol and may add further packets e.g., TCP Syn/Ack packets. The current implementations pass the generated packets to the underlying Link and store information like connected Ports, used Protocols and a user-defined labels, e.g. malicious.

Links model protocols of lower OSI levels down to physical connections, which are used to connect Smart Devices. For instance, the Link-interface can be used to model wired or wireless communication standards like Ethernet or Wifi. It defines the packet loss and transmission delay of Connections and Protocols, which use the respective Link. A Link can connect multiple Smart Devices simultaneously. The current implementation abstractly models the lower layers but does not add additional packets or wrappers. The base class for Packets encompasses a source, destination, timestamp, and a payload. Additionally, methods for exporting the packet in byte format and a human-readable format are provided:

[MQTT: PUBLISH; time:1337; source:SmartDoor:1883; destination:Broker:1883; topic:/home/doorOpen:true]

Subclasses of Packets can model packets down to the byte level and add further details. For example, the MQTT Protocol adds MQTT headers, flags, and topics to the packets.

\subsection{User Interface}

The main purpose of the framework is to train and evaluate datadriven algorithms in a modeled network environment. For this, a visualization of the network model, devices and their connections supports users in quickly making adaptions to scenarios and the mechanisms, which improves the quality of the evaluation process. Figure 2 shows the main view of the simulator and the corresponding network tree of an exemplary network. The VisualizationPanel represents the main modeling canvas and visualizes the network model. The basic building blocks of the model are Smart Devices (e.g., WiFi router), which are represented as circles. Links between Smart Devices are visualized as fractions of colored borders that are placed around the devices. Common Links between Smart Devices can be identified by the same color and the same relative location of the border. For instance, the ZigBee- and WiFi-Router share a Link indicated by the black border at the top-right position. Connections and their encapsulated Protocols are visualized by green lines between devices. Users can interact and edit all Smart Devices, Connections, and Links. For instance, users can change the timings of Ports during the simulation and Smart Devices can be added or removed. This enables flexible modeling of scenarios and investigating changing conditions at runtime. The NetworkTreePanel represents the network as a tree-structure to highlight interdependencies and capsuled configurations. Here, Links are organized on the first level, Connections on the second level, and Smart Devices on the third one. This structure allows navigating through large networks easily; Moreover, using this representation, parts of the network can be hidden on the VisualizationPanel. This reduces the number of visible devices and improves visibility and enables users to focus on specific parts of the network. The menu bar at the top contains various menus for configuring individual parts of the framework and for simulation purposes. The simulation menu can be used to configure simulation parameters like start time, end time, and interval duration and to export configurations and results into files. Another important menu aspect related to simulation is the algorithm menu, which provides capabilities for loading Javabased algorithms and execute them within the currently modeled network.

\subsection{Extensible Design}

An important feature of the framework is the extensible design that allows integrating customized Protocols, Smart Devices algorithms to be evaluated. The framework supports compiling and importing Java files at runtime, which provides several benefits from the perspective of extensibility and evaluation capabilities: 
- Easy import of customized implementations of Protocols, Connections, and Links.

- Support for various types of (new) Smart Devices.

- Easy valuation of algorithms for interacting with the modeled network.

The framework currently supports two groups of algorithms, namely Environment Manipulation Algorithms and Data Analysis Algorithms (DAAs). The first type allows scripting simulation scenarios to adapt the simulated model to the preferences of the user (e.g., replicating properties of a specific real-world network). DAAs represent algorithms that process network data during the simulation, like training and evaluating anomaly detection mechanisms. The different algorithms are explained below in more detail.

Environment Manipulation Algorithm. Representing the behavior of a target network within a simulated environment is a challenging task. Especially challenging are effects that emerge from the behavior of heterogeneous devices, their configuration, and environmental influences. For instance, a router could block certain ports, the WiFi signal could be distorted due to bad topological setups or environmental changes (e.g., people moving). To enable the representation of such peculiarities Environmental Manipulation Algorithms allow adapting simulations during run-time. Among these adaptions are adding, configuring, and modifying parts of the network model like Smart Devices, Connections, and Links as well as manipulating transmitted packages.

Data Analysis Algorithm (DAA). DAAs are data-driven approaches that are to be trained and/or evaluated within the framework. In contrast to the Environment Manipulation Algorithms, they do not have full access to the model but can only operate on applicationlayer packets that are generated during the simulation. This access is customizable, taking into account that algorithms can have a global view on the network, or are limited to the scope of individual Smart Devices or Connections. This allows simulating scenarios of parallel networks or remote devices, where the observable scope is limited. DAAs can define a training- and a testing-mode to distinguish between two main phases of their execution. The training-mode enables live-training, where observed packets (during the simulation) are used for training. In contrast, the testing-mode activates the intended functions of the algorithms (e.g., anomaly detection) and treats simulated packets as input. DAAs can switch between the two modes at any point in time and their view on the network can be adjusted. This supports simulating situations where nodes, responsible for observing parts of a network, are failing and the algorithm receives a reduced set of information.

\subsection{Simulation Capabilities}

This subsection introduces the simulation capabilities of the framework. The framework combines discrete-event simulation and a sliced-time approach for the execution of algorithms and the export of packets. The main parameters relevant for the simulation are the current timestep, interval duration, start time, and end time. Parameters can be freely configured and define start- and stop-time of a simulation as well as the duration of an interval. An interval specifies the number of time steps for which the network simulation is conducted prior to any modification or analysis processes.

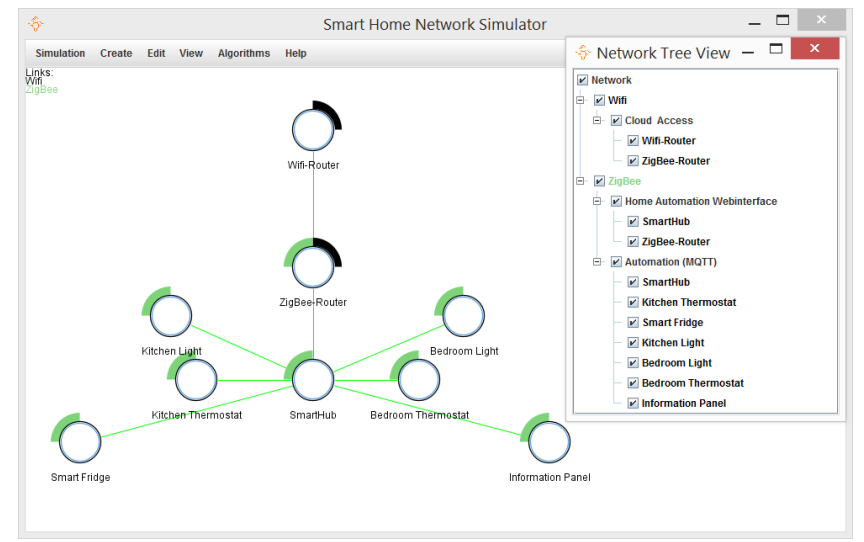

Figure 2: Test scenario within the framework

Afterwards, the Environment Manipulation Algorithms are executed followed by the DAAs. The DAAs will receive all packets that are visible within their views that were transmitted within the specified interval.

3.4.1 Network Simulation. The network uses events that are organized in a global event queue to ensure correctness of their order. Events can be added to the queue by all components of the network at arbitrary points in time. For instance, Protocols can add events that will be executed at later points in time, like value updates as a reaction to incoming packets. The simulation order is indicated by Arabic numbers presented in Fig. 1. In step 1), the Port simulation is triggered. Subsequently, 2) the Port triggers the Protocol execution to generate network packets. In step 3) these packets are forwarded to the underlying Connection. Similarly, Step 4) uses the underlying Link to encapsulate packets. Finally, steps 5) and 6) represent the use of the encapsulated packets for data exports or within algorithms. If multiple events are scheduled at the same time, the scheduler operates in a first-in-first-out (FIFO) fashion.

\section{CASE STUDY}

In this section, we present the capabilities of the framework using a smart home scenario. First, the example network is introduced. Second, the details of the conducted simulation are explained. Finally, the results for using the generated packets for training and testing an anomaly detection algorithm are discussed.

\subsection{Scenario description}

The scenario depicted in Fig. 2 represents a smart-home setup. The components are an IoT thermostat, a smart light-bulb, an information panel, a smart fridge, and two routers that connect the smart home to the Internet and provide a ZigBee protocol and Wifi connection. A smart hub manages the home automation.

For establishing network communication the MQTT protocol is used. The protocol implements the publish-subscribe pattern based on brokers for packet redistribution, publishers for sending packets, and subscribers as packet receivers. In our example, the smart hub acts as the broker, the information panel is a subscriber, and light-bulbs, smart fridge, and thermostats are publishers. 
The sending frequencies for packets are modeled using normal distributions with varying parameter settings. For the case study, the mean and standard deviation are empirically chosen to represent typical IoT device behavior. Messages are sent in intervals of 15 seconds on average and a standard deviation of 0.5 seconds. Cloud access is available every second, with a standard deviation of $0.3 \mathrm{~s}$ and the web interfaces for devices are available every $5 \mathrm{~s}$ with a standard deviation of $3 \mathrm{~s}$. It is assumed that initially all devices work as intended such that no abnormal behavior is present.

\subsection{Simulation Setup}

The focus of the evaluation lies on the live training and evaluation capabilities of the framework. The "offline" dataset generation functionality (i.e., generate and export a dataset) relies on the live-data generation and is therefore omitted in this evaluation.

Two core-scenarios are considered: First, a Denial of Service scenario, where a Denial of Service (DoS) and Distributed Denial of Service (DDoS) attack are conducted. Second, an Integrity Tamper scenario, where abnormal measurement data for a sensor is introduced. The anomaly detection mechanism is based on an adaption of the K-means algorithm presented by Münz et al. [13]. It receives full access to all packets that are transmitted in the network. The goal of this case study is to showcase the analysis functionality of the framework. We show, that an initial configuration of the algorithm is not well-suited to detect all introduced anomalies but the capabilities of the framework enable users to quickly adapt, re-train and evaluate algorithms to successfully detect all anomalies.

The network is simulated for 28 virtual hours. 24 hours are used for the training the K-Means algorithm with full access to the packet traffic within the network. After 24 hours, the anomaly detection phase starts and the algorithm aims at detecting anomalies within the packet traffic. All anomalies are injected individually (i.e., no simultaneous occurring anomalies are considered) and are executed for a duration of one hour. The first anomaly is a DDoS-attack, where all devices of the ZigBee network target the smart hub. They sent packets disguised in the MQTT format with a high frequency of $1.5 \mathrm{~s}$. Subsequently, a DoS attack is injected, whereby the kitchen light-bulb sends 250 packets per second to the fridge. This sending behavior was empirically defined to be not stealthy and represents a thousandfold increase compared to the normal sending behavior. The second attack scenario introduces modified meter readings into the network. These readings mimic an unexpected defrosting of the smart fridge. Temperature values are increased from a normal range between -6 and -4 degrees to an abnormal range of 18 to 24 degrees. The values are published regularly every $15 \mathrm{~s}$. All evaluations are conducted on a notebook running Windows 8.1 on an Intel $17-4710 \mathrm{HQ}$ CPU at $2.50 \mathrm{GHz}$ and $8 \mathrm{~GB}$ RAM.

\subsection{Results}

Table 1 presents the results of the initial application (prior to adjustments and re-training) of the K-Means algorithm. Important parameters are the number of sent packets per task (\#Packets) and the execution time for the packet generation (Elapsed). For assessing the performance of the K-means algorithm, the True Positives (TPs), True Negatives (TNs), False Positives (FPs), False Negatives (FNs), and the execution times are listed.

\begin{tabular}{lrrrrrr}
\hline Task & \#Packets & TP & TN & FP & FN & Elapsed \\
\hline Training Traffic & 342452 & - & - & - & - & $489 \mathrm{~ms}$ \\
\hline K-Means Training & - & - & - & - & - & $18481 \mathrm{~ms}$ \\
\hline \hline Normal Traffic & 14398 & 0 & 14398 & 0 & 0 & $35 \mathrm{~ms}$ \\
\hline Attack (DDoS) & 28978 & 14620 & 14358 & 0 & 0 & $175 \mathrm{~ms}$ \\
\hline Attack (DoS) & 914267 & 899999 & 14268 & 0 & 0 & $1826 \mathrm{~ms}$ \\
\hline Value anomaly & 14354 & 0 & 14268 & 0 & 86 & $40 \mathrm{~ms}$ \\
\hline
\end{tabular}

Table 1: Results of the evaluation presenting the different tasks, numbers of classified packets, and runtimes.

The simulation of the network and the data generation takes $489 \mathrm{~ms}$. During this time, 342,452 packets are generated. Approximately $18.5 \mathrm{~s}$ are required for the training of the K-means algorithm. For the remaining tasks, the simulation time depends linearly on the number of generated packets. Table 1 presents the detailed results.

The Normal Traffic phase is assumed to not contain any anomalies. The 14,398 TNs are the normal traffic packets sent during the specified one hour of conducted attacks. Variations within the number of packets are caused by the randomness of the distribution functions used for generating traffic of the different devices.

Both DoS and DDoS attacks are detected by the initial configuration of the K-means. The DDoS attack is detected by identifying 14,620 packets as TPs and 14,358 packets as TNs out of 28,978 generated packets. The FPs and FNs are 0. Consequently, the True Positive Rate (TPR) is 1. Abnormal packets are classified correctly, as sending frequency and ports deviate from normal traffic and the learned boundaries of the K-Means algorithm are small. The DoS attack is detected by analyzing 914,267 packets and the algorithm again reaches a TPR of 1 with 899,999 TPs and 14,268 TNs.

The value anomalies are not detected by the algorithm. In this case, 14,354 packets are generated, out of which 14,268 are correctly identified as TNs and 86 are FNs. This can be attributed to the generalization of the K-Means clusters such that the relatively small deviations in the payload values can not be detected.

To improve this performance, we adapt the algorithm and conduct a second evaluation run. The number of clusters is incrementally increased to 34 (from the initial 13). Additionally, the initialization method is changed to use the farthest instances as first clusters (a random initialization was used before). Table 2 shows the results for the second simulation run.

We want to emphasize that this adaption is easily possible using the capabilities of the framework. In a real-world environment, the same evaluation takes multiple hours or days to identify a suitable parameter setting that detects all anomalies present in a scenario.

The simulation time is slightly increased compared to the initial run but the training of the algorithm is faster with only 4.4 seconds, as the clustering algorithm converges more quickly. In this second run, the algorithm successfully identifies the Integrity Tamper anomalies and achieves a TPR of 1 for all attack scenarios. 


\begin{tabular}{lrrrrrr}
\hline Task & \#Packets & TP & TN & FP & FN & Elapsed \\
\hline Training Traffic & 343712 & - & - & - & - & $624 \mathrm{~ms}$ \\
\hline K-Means Training & - & - & - & - & - & $4393 \mathrm{~ms}$ \\
\hline \hline Normal Traffic & 14402 & 0 & 14402 & 0 & 0 & $95 \mathrm{~ms}$ \\
\hline Attack (DDoS) & 38214 & 23968 & 14246 & 0 & 0 & $114 \mathrm{~ms}$ \\
\hline Attack (DoS) & 914399 & 899999 & 14400 & 0 & 0 & $3046 \mathrm{~ms}$ \\
\hline Value anomaly & 14388 & 164 & 14224 & 0 & 0 & $61 \mathrm{~ms}$ \\
\hline
\end{tabular}

Table 2: Results of the second evaluation representing the different tasks, numbers of classified packets, and runtimes.

The case study shows how a user can leverage the framework to perform experiments for evaluating DAAs that operate on packetlevel network traffic. The results show that the framework is capable of generating large amounts of data quickly and provide these to DAAs for their evaluation. Simulated networks can be arbitrarily modified at runtime, enabling the introduction of various anomalies, as shown by the introduction of a DDoS, DoS, and Integrity Tamper attack. The case study also highlights the capabilities of the framework to enable quick parameter adaptions, followed by redeployment of modified approaches and facilitates quick and easy re-training. With this, various parameter settings for the DAAs can be evaluated quickly to find optimal configurations for the desired performance criteria (e.g., all types of anomalies found).

\section{CONCLUSION}

In this work, we presented a framework for supporting the development and evaluation process of data-driven algorithms in a virtual environment. The framework provides intensive packet-level data simulation capabilities, which can be leveraged for generating and exporting standalone datasets and for live training and evaluation. For the latter, the framework enables the virtual deployment of so-called Data Analysis Algorithms (DAAs), which can access the simulated data and use it for training and testing purposes.

The work is evaluated by simulating an exemplary smart home environment that communicates via the MQTT protocol. Various anomalies, like Denial of Service (DoS), Distributed Denial of Service (DDoS) attacks, and measurement value manipulations, are introduced into the simulation. An anomaly detection approach is trained and evaluated on the modeled network. The results show that the framework can be used to support the training and evaluation of data-driven algorithms and speed up their deployment.

\section{ACKNOWLEDGMENTS}

This work is funded by the Software Campus projects "ADRAS" and "ALIEN4IoT", under the German Federal Ministry of Education and Research (BMBF) grant no. 01IS17050 and jointly by BMBF and the Hessen State Ministry for Higher Education, Research and the Arts in support of the National Research Center for Applied Cybersecurity ATHENE

\section{REFERENCES}

[1] Cisco Networking Academy. 2019. Cisco Packet Tracer. Retrieved 14th April 2020 from https://www.netacad.com/courses/packet-tracer
[2] Nasser Alshammari, Talal Alshammari, Mohamed Sedky, Justin Champion, and Carolin Bauer. 2017. OpenSHS: Open Smart Home Simulator. Sensors 17, 5 (2017). https://doi.org/10.3390/s17051003

[3] Manos Antonakakis, Tim April, Michael Bailey, Matt Bernhard, Elie Bursztein, Jaime Cochran, Zakir Durumeric, J Alex Halderman, Luca Invernizzi, Michalis Kallitsis, et al. 2017. Understanding the mirai botnet. In 26th \{USENIX\} Security Symposium (\{USENIX\} Security 17). 1093-1110.

[4] Pat Bosshart, Dan Daly, Glen Gibb, Martin Izzard, Nick McKeown, Jennifer Rexford, Cole Schlesinger, Dan Talayco, Amin Vahdat, George Varghese, and David Walker. 2014. P4: Programming Protocol-independent Packet Processors. SIGCOMM Comput. Commun. Rev. 44, 3 (July 2014), 87-95. https://doi.org/10. $1145 / 2656877.2656890$

[5] Kevin Bouchard, Amir Ajroud, Bruno Bouchard, and Abdenour Bouzouane. 2010. SIMACT: A 3D Open Source Smart Home Simulator for Activity Recognition. In Advances in Computer Science and Information Technology, Tai-hoon Kim and Hojjat Adeli (Eds.). Springer Berlin Heidelberg, Berlin, Heidelberg, 524-533.

[6] Julien Bruneau, Wilfried Jouve, and Charles Consel. 2009. DiaSim: A Parameterized Simulator for Pervasive Computing Applications. In 6th International Conference on Mobile and Ubiquitous Systems: Computing, Networking and Services (Mobiquitous'09). IEEE, Toronto, Canada. https://hal.inria.fr/inria-00403421

[7] Varun Chandola, Arindam Banerjee, and Vipin Kumar. 2009. Anomaly detection: A survey. ACM computing surveys (CSUR) 41, 3 (2009), 15.

[8] Carlos Garcia Cordero, Emmanouil Vasilomanolakis, Nikolay Milanov, Christian Koch, David Hausheer, and Max Mühlhäuser. 2015. ID2T: a DIY dataset creation toolkit for Intrusion Detection Systems. In 2015 IEEE Conference on Communications and Network Security (CNS). IEEE, 739-740.

[9] Chengze Fan, Jun Bi, Yu Zhou, Cheng Zhang, and Haisu Yu. 2017. NS4: A P4driven Network Simulator. 105-107. https://doi.org/10.1145/3123878.3132002

[10] Ostinato Packet Generator. 2019. Ostinato Packet Generator. Retrieved 14th April 2020 from https://ostinato.org

[11] Abdelsalam Helal, Jaewoong Lee, Shantonu Hossain, Eunju Kim, Hani Hagras, and Diane J. Cook. 2011. Persim - Simulator for Human Activities in Pervasive Spaces. 2011 Seventh International Conference on Intelligent Environments (2011), 192-199.

[12] S Mehta, KS Kwak, and Najnin Sulatan. 2010. Network and system simulation tools for next generation networks: a case study. INTECH Open Access Publisher.

[13] Gerhard Münz, Sa Li, and Georg Carle. 2007. Traffic Anomaly Detection Using KMeans Clustering. In In GI/ITG Workshop MMBnet.

[14] Nam: Network Animator 2002. Nam: Network Animator. Retrieved 14th April 2020 from https://www.isi.edu/nsnam/nam/

[15] Network Simulator 2 2011. The Network Simulator - ns-2. Retrieved 14th April 2020 from http://nsnam.sourceforge.net/wiki/index.php/User_Information

[16] Hiroshi Nishikawa, Shinya Yamamoto, Morihiko Tamai, Kouji Nishigaki, Tomoya Kitani, Naoki Shibata, Keiichi Yasumoto, and Minoru Ito. 2006. UbiREAL: Realistic Smartspace Simulator for Systematic Testing. In UbiComp 2006: Ubiquitous Computing, Paul Dourish and Adrian Friday (Eds.). Springer Berlin Heidelberg, Berlin, Heidelberg, 459-476.

[17] Sutharshan Rajasegarar, Christopher Leckie, and Marimuthu Palaniswami. 2008. Anomaly detection in wireless sensor networks. IEEE Wireless Communications 15, 4 (2008), 34-40.

[18] George F. Riley and Thomas R. Henderson. 2010. The ns-3 Network Simulator. Springer Berlin Heidelberg, Berlin, Heidelberg, 15-34. https://doi.org/10.1007/ 978-3-642-12331-3_2

[19] Avneet Kaur Saluja, Sweta A Dargad, and Krupali Mistry. 2017. A Detailed Analogy of Network Simulators - NS1, NS2, NS3 and NS4. http://www.ijfrcsce.org. International fournal on Future Revolution in Computer Science \& Communication Engineering 3 (2017), 291-295.

[20] SimpleSoft. 2019. Simple IoT Simulator by SimpleSoft. Retrieved 14th April 2020 from http://www.smplsft.com/SimpleIoTSimulator.html

[21] Smart Home Network Simulator [n. d.]. Smart Home Network Simulator. https://anonymous.4open.science/r/360add55-49f8-40ba-b6c6-91a2dfebcfe5/. Online; accessed 14th April 2020

[22] Jonathan Synnott, Chris Nugent, and Paul Jeffers. 2015. Simulation of Smart Home Activity Datasets. Sensors 15, 6 (2015), 14162-14179. https://doi.org/10. 3390/s150614162

[23] Tetcos. 2019. NetSim. Retrieved 14th April 2020 from https://tetcos.com/

[24] Chih-Fong Tsai, Yu-Feng Hsu, Chia-Ying Lin, and Wei-Yang Lin. 2009. Intrusion detection by machine learning: A review. expert systems with applications 36,10 (2009), 11994-12000.

[25] András Varga and Rudolf Hornig. 2008. An overview of the OMNeT++ simulation environment. In Proceedings of the 1st international conference on Simulation tools and techniques for communications, networks and systems \& workshops. ICST (Institute for Computer Sciences, Social-Informatics and ..., 60.

[26] C. Welsh. 2013. GNS3 Network Simulation Guide. Packt Publishing. https: //books.google.de/books?id=RcgUngEACAAJ

[27] Ian H. Witten, Eibe Frank, Mark A. Hall, and Christopher J. Pal. 2016. Data Mining, Fourth Edition: Practical Machine Learning Tools and Techniques (4th ed.). Morgan Kaufmann Publishers Inc., San Francisco, CA, USA. 